\title{
MINIMAL 2-DOMINATING SETS IN TREES *
}

\author{
MARCIN KRZYWKOWSKI ${ }^{1}$
}

\begin{abstract}
We provide an algorithm for listing all minimal 2 -dominating sets of a tree of order $n$ in time $\mathcal{O}\left(1.3248^{n}\right)$. This implies that every tree has at most $1.3248^{n}$ minimal 2-dominating sets. We also show that this bound is tight.
\end{abstract}

Mathematics Subject Classification. 05C05, 05C69, 05C85, 68R10, 68W05.

\section{INTRODUCTION}

Let $G=(V, E)$ be a graph. The order of a graph is the number of its vertices. By the neighborhood of a vertex $v$ of $G$ we mean the set $N_{G}(v)=\{u$ $\in V(G): u v \in E(G)\}$. The degree of a vertex $v$, denoted by $d_{G}(v)$, is the cardinality of its neighborhood. By a leaf we mean a vertex of degree one, while a support vertex is a vertex adjacent to a leaf. The distance between two vertices of a graph is the number of edges in a shortest path connecting them. The eccentricity of a vertex is the greatest distance between it and any other vertex. The diameter of a graph $G$, denoted by $\operatorname{diam}(G)$, is the maximum eccentricity among all vertices of $G$. By $P_{n}$ we denote a path on $n$ vertices. By a star we mean a connected graph in which exactly one vertex has degree greater than one.

A subset $D \subseteq V(G)$ is a dominating set of $G$ if every vertex of $V(G) \backslash D$ has a neighbor in $D$, while it is a 2-dominating set of $G$ if every vertex of $V(G) \backslash D$ has at least two neighbors in $D$. A dominating (2-dominating, respectively) set $D$ is minimal if no proper subset of $D$ is a dominating (2-dominating, respectively) set of $G$.

Keywords and phrases. Domination, 2-domination, minimal 2-dominating set, tree, counting, exact exponential algorithm, listing algorithm.

* The research was supported by the Polish National Science Centre grant 2011/02/A/ST6/00201.

1 Faculty of Electronics, Telecommunications and Informatics, Gdańsk University of Technology, Narutowicza 11/12, 80-233 Gdańsk, Poland. marcin.krzywkowski@gmail.com 
A minimal 2-dominating set is abbreviated as $\mathrm{m} 2 \mathrm{ds}$. Note that 2-domination is a type of multiple domination in which each vertex, which is not in the dominating set, is dominated at least $k$ times for a fixed positive integer $k$. Multiple domination was introduced by Fink and Jacobson [7], and further studied for example in $[2,10,17]$. For a comprehensive survey of domination in graphs, see $[11,12]$.

Observation 1.1. Every leaf of a graph $G$ is in every 2-dominating set of $G$.

One of the typical questions in graph theory is how many subgraphs of a given property can a graph on $n$ vertices have. For example, the famous Moon and Moser theorem [16] says that every graph on $n$ vertices has at most $3^{n / 3}$ maximal independent sets.

Combinatorial bounds are of interest not only on their own, but also because they are used for algorithm design as well. Lawler [15] used the Moon-Moser bound on the number of maximal independent sets to construct an $(1+\sqrt[3]{3})^{n} \cdot n^{\mathcal{O}(1)}$ time graph coloring algorithm, which was the fastest one known for twenty-five years. In 2003 Eppstein [6] reduced the running time of a graph coloring to $\mathcal{O}\left(2.4151^{n}\right)$. In 2006 the running time was reduced $[1,14]$ to $\mathcal{O}\left(2^{n}\right)$. For an overview of the field, see $[9]$.

Fomin et al. [8] constructed an algorithm for listing all minimal dominating sets of a graph on $n$ vertices in time $\mathcal{O}\left(1.7159^{n}\right)$. There were also given graphs $(n / 6$ disjoint copies of the octahedron) having $15^{n / 6} \approx 1.5704^{n}$ minimal dominating sets. This establishes a lower bound on the running time of an algorithm for listing all minimal dominating sets of a given graph.

The number of maximal independent sets in trees was investigated in [18]. Couturier et al. [5] considered minimal dominating sets in various classes of graphs. The authors of [13] investigated the enumeration of minimal dominating sets in graphs.

Bród and Skupień [3] gave bounds on the number of dominating sets of a tree. They also characterized the extremal trees. The authors of [4] investigated the number of minimal dominating sets in trees containing all leaves.

We provide an algorithm for listing all minimal 2-dominating sets of a tree of order $n$ in time $\mathcal{O}\left(1.3248^{n}\right)$. This implies that every tree has at most $1.3248^{n}$ minimal 2-dominating sets. We also show that this bound is tight.

\section{Listing ALGORITHM}

In this section we describe a recursive algorithm which lists all minimal 2 -domina-ting sets of a given input tree $T$. The iterator of the solutions is denoted by $\mathcal{F}(T)$.

\section{Algorithm}

Notice that the diameter of a tree can be easily determined in a polynomial time. 
Let $T$ be a tree. If $\operatorname{diam}(T)=0$, then $T=P_{1}=v_{1}$. Let $\mathcal{F}(T)=\left\{\left\{v_{1}\right\}\right\}$. If $\operatorname{diam}(T)=1$, then $T=P_{2}=v_{1} v_{2}$. Let $\mathcal{F}(T)=\left\{\left\{v_{1}, v_{2}\right\}\right\}$. If $\operatorname{diam}(T)=2$, then $T$ is a star. By $x$ we denote the support vertex of $T$. Let $\mathcal{F}(T)=\{V(T) \backslash\{x\}\}$.

Now consider trees $T$ with $\operatorname{diam}(T) \geq 3$. Thus the order $n$ of the tree $T$ is at least four.

If some support vertex of $T$, say $x$, is adjacent to at least three leaves (we denote one of them by $y$ ), then let $T^{\prime}=T-y$ and

$$
\mathcal{F}(T)=\left\{D^{\prime} \cup\{y\}: D^{\prime} \in \mathcal{F}\left(T^{\prime}\right)\right\}
$$

Now consider trees $T$, in which every support vertex is adjacent to at most two leaves. The tree $T$ can easily be rooted at a vertex $r$ of maximum eccentricity $\operatorname{diam}(T)$ in polynomial time. A leaf, say $t$, at maximum distance from $r$, can also be easily computed in polynomial time. Let $v$ denote the parent of $t$ and let $u$ denote the parent of $v$ in the rooted tree. If $\operatorname{diam}(T) \geq 4$, then let $w$ denote the parent of $u$. By $T_{x}$ we denote the subtree induced by a vertex $x$ and its descendants in the rooted tree $T$.

If $d_{T}(v)=3$, then by $a$ we denote the leaf adjacent to $v$ and different from $t$. Let $T^{\prime}=T-T_{v}$ and $T^{\prime \prime}=T-t-a$, and let $\mathcal{F}(T)$ be as follows,

$$
\begin{aligned}
& \left\{D^{\prime} \cup\{t, a\}: D^{\prime} \in \mathcal{F}\left(T^{\prime}\right)\right\} \\
\cup & \left\{D^{\prime \prime} \cup\{t, a\}: D^{\prime \prime} \in \mathcal{F}\left(T^{\prime \prime}\right) \text { and } D^{\prime \prime} \backslash\{v\} \notin \mathcal{F}\left(T^{\prime}\right)\right\} .
\end{aligned}
$$

If $d_{T}(v)=2$ and $d_{T}(u) \geq 3$, then let $T^{\prime}=T-T_{v}, T^{\prime \prime}=T-T_{u}$, and

$$
\mathcal{F}(T)=\left\{D^{\prime} \cup\{t\}: u \in D^{\prime} \in \mathcal{F}\left(T^{\prime}\right)\right\} \cup\left\{D^{\prime \prime} \cup V\left(T_{u}\right) \backslash\{u\}: D^{\prime \prime} \in \mathcal{F}\left(T^{\prime \prime}\right)\right\} .
$$

If $d_{T}(v)=d_{T}(u)=2$, then let $T^{\prime}=T-T_{v}, T^{\prime \prime}=T-T_{u}$, and

$$
\mathcal{F}(T)=\left\{D^{\prime} \cup\{t\}: D^{\prime} \in \mathcal{F}\left(T^{\prime}\right)\right\} \cup\left\{D^{\prime \prime} \cup\{v, t\}: w \in D^{\prime \prime} \in \mathcal{F}\left(T^{\prime \prime}\right)\right\} .
$$

\section{Bounding the Number of Minimal 2-Dominating SETS}

Now we prove that the running time of the algorithm from the previous section is $\mathcal{O}\left(1.3248^{n}\right)$.

Theorem 3.1. For every tree $T$ of order $n$, the algorithm from the previous section lists all minimal 2-dominating sets in time $\mathcal{O}\left(1.3248^{n}\right)$.

Proof. We prove that the running time of the algorithm is $\mathcal{O}\left(1.3248^{n}\right)$. Moreover, we prove that the number of minimal 2-dominating sets of $T$ is at most $\alpha^{n}$, where $\alpha \approx 1.3248$ is the positive solution of the equation $x^{3}-x-1=0$.

We proceed by induction on the number $n$ of vertices of a tree $T$. If $\operatorname{diam}(T)$ $=0$, then $T=P_{1}=v_{1}$. Obviously, $\left\{v_{1}\right\}$ is the only m2ds of the path $P_{1}$. We have $n=1$ and $|\mathcal{F}(T)|=1$. Obviously, $1<\alpha$. If $\operatorname{diam}(T)=1$, then $T=P_{2}=v_{1} v_{2}$. It is easy to see that $\left\{v_{1}, v_{2}\right\}$ is the only m2ds of the path $P_{2}$. We have $n=2$ and 
$|\mathcal{F}(T)|=1$. Obviously, $1<\alpha^{2}$. If $\operatorname{diam}(T)=2$, then $T$ is a star. By $x$ we denote the support vertex of $T$. It is easy to observe that $V(T) \backslash\{x\}$ is the only m2ds of the tree $T$. We have $n \geq 3$ and $|\mathcal{F}(T)|=1$. Obviously, $1<\alpha^{n}$.

Now assume that $\operatorname{diam}(T) \geq 3$. Thus the order $n$ of the tree $T$ is at least four. The results we obtain by the induction on the number $n$. Assume that they are true for every tree $T^{\prime}$ of order $n^{\prime}<n$.

First assume that some support vertex of $T$, say $x$, is adjacent to at least three leaves. Let $y$ be a leaf adjacent to $x$. Let $T^{\prime}=T-y$. Let $D^{\prime}$ be a m2ds of the tree $T^{\prime}$. Obviously, $D^{\prime} \cup\{y\}$ is an $\mathrm{m} 2 \mathrm{ds}$ of $T$. Thus all elements of $\mathcal{F}(T)$ are minimal 2-dominating sets of the tree $T$. Now let $D$ be any m2ds of the tree $T$. By Observation 1.1 we have $y \in D$. Let us observe that $D \backslash\{y\}$ is an $\mathrm{m} 2 \mathrm{ds}$ of the tree $T^{\prime}$ as the vertex $x$ is still dominated at least twice. By the inductive hypothesis we have $D \backslash\{y\} \in \mathcal{F}\left(T^{\prime}\right)$. Therefore $\mathcal{F}(T)$ contains all minimal 2-dominating sets of the tree $T$. Now we get $|\mathcal{F}(T)|=\left|\mathcal{F}\left(T^{\prime}\right)\right| \leq \alpha^{n-1}<\alpha^{n}$. Henceforth, we can assume that every support vertex of $T$ is adjacent to at most two leaves.

We now root $T$ at a vertex $r$ of maximum eccentricity $\operatorname{diam}(T)$. Let $t$ be a leaf at maximum distance from $r, v$ be the parent of $t$, and $u$ be the parent of $v$ in the rooted tree. If $\operatorname{diam}(T) \geq 4$, then let $w$ be the parent of $u$. By $T_{x}$ we denote the subtree induced by a vertex $x$ and its descendants in the rooted tree $T$.

Assume that $d_{T}(v)=3$. By $a$ we denote the leaf adjacent to $v$ and different from $t$. Let $T^{\prime}=T-T_{v}$ and $T^{\prime \prime}=T-t-a$. Let us observe that all elements of $\mathcal{F}(T)$ are minimal 2-dominating sets of the tree $T$. Now let $D$ be any $\mathrm{m} 2 \mathrm{ds}$ of the tree $T$. By Observation 1.1 we have $t, a \in D$. If $v \notin D$, then observe that $D \backslash\{t, a\}$ is an $\mathrm{m} 2 \mathrm{ds}$ of the tree $T^{\prime}$. By the inductive hypothesis we have $D \backslash\{t, a\} \in \mathcal{F}\left(T^{\prime}\right)$. Now assume that $v \in D$. Let us observe that $D \backslash\{t, a\}$ is an m2ds of the tree $T^{\prime \prime}$. By the inductive hypothesis we have $D \backslash\{t, a\} \in \mathcal{F}\left(T^{\prime \prime}\right)$. The set $D \backslash\{v, t, a\}$ is not an m2ds of the tree $T^{\prime}$, otherwise $D \backslash\{v\}$ is a 2dominating set of the tree $T$, a contradiction to the minimality of $D$. By the inductive hypothesis we have $D \backslash\{v, t, a\} \notin \mathcal{F}\left(T^{\prime}\right)$. Therefore $\mathcal{F}(T)$ contains all minimal 2-dominating sets of the tree $T$. Now we get $|\mathcal{F}(T)|=\left|\mathcal{F}\left(T^{\prime}\right)\right|+\mid D^{\prime \prime}$ $\in \mathcal{F}\left(T^{\prime \prime}\right): D^{\prime \prime} \backslash\{v\} \notin \mathcal{F}\left(T^{\prime}\right)|\leq| \mathcal{F}\left(T^{\prime}\right)|+| \mathcal{F}\left(T^{\prime \prime}\right) \mid \leq \alpha^{n-3}+\alpha^{n-2}=\alpha^{n-3}(\alpha+1)$ $=\alpha^{n-3} \cdot \alpha^{3}=\alpha^{n}$.

Now assume that $d_{T}(v)=2$. Assume that $d_{T}(u) \geq 3$. Let $T^{\prime}=T-T_{v}$ and $T^{\prime \prime}=T-T_{u}$. Let us observe that all elements of $\mathcal{F}(T)$ are minimal 2-dominating sets of the tree $T$. Now let $D$ be any m2ds of the tree $T$. By Observation 1.1 we have $t \in D$. If $v \notin D$, then $u \in D$ as the vertex $v$ has to be dominated twice. Observe that $D \backslash\{t\}$ is an m2ds of the tree $T^{\prime}$. By the inductive hypothesis we have $D \backslash\{t\} \in \mathcal{F}\left(T^{\prime}\right)$. Now assume that $v \in D$. We have $u \notin D$, otherwise $D \backslash\{v\}$ is a 2-dominating set of the tree $T$, a contradiction to the minimality of $D$. Observe that $D \cap V\left(T^{\prime \prime}\right)$ is an $\mathrm{m} 2 \mathrm{ds}$ of the tree $T^{\prime \prime}$. By the inductive hypothesis we have $D \cap V\left(T^{\prime \prime}\right) \in \mathcal{F}\left(T^{\prime \prime}\right)$. Therefore $\mathcal{F}(T)$ contains all minimal 2-dominating sets of the tree $T$. Now we get $|\mathcal{F}(T)| \leq\left|\mathcal{F}\left(T^{\prime}\right)\right|+\left|\mathcal{F}\left(T^{\prime \prime}\right)\right| \leq \alpha^{n-2}+\alpha^{n-3}=\alpha^{n-3}(\alpha+1)=$ $\alpha^{n-3} \cdot \alpha^{3}=\alpha^{n}$. 
Now assume that $d_{T}(u)=2$. Let $T^{\prime}=T-T_{v}$ and $T^{\prime \prime}=T-T_{u}$. Let us observe that all elements of $\mathcal{F}(T)$ are minimal 2-dominating sets of the tree $T$. Now let $D$ be any $\mathrm{m} 2 \mathrm{ds}$ of the tree $T$. By Observation 1.1 we have $t \in D$. If $v \notin D$, then observe that $D \backslash\{t\}$ is an $\mathrm{m} 2$ ds of the tree $T^{\prime}$. By the inductive hypothesis we have $D \backslash\{t\} \in \mathcal{F}\left(T^{\prime}\right)$. Now assume that $v \in D$. We have $u \notin D$, otherwise $D \backslash\{v\}$ is a 2-dominating set of the tree $T$, a contradiction to the minimality of $D$. Moreover, we have $w \in D$ as the vertex $u$ has to be dominated twice. Observe that $D \backslash\{v, t\}$ is an m2ds of the tree $T^{\prime \prime}$. By the inductive hypothesis we have $D \backslash\{v, t\} \in \mathcal{F}\left(T^{\prime \prime}\right)$. Therefore $\mathcal{F}(T)$ contains all minimal 2-dominating sets of the tree $T$. Now we get $|\mathcal{F}(T)| \leq\left|\mathcal{F}\left(T^{\prime}\right)\right|+\left|\mathcal{F}\left(T^{\prime \prime}\right)\right| \leq \alpha^{n-2}+\alpha^{n-3}=\alpha^{n-3}(\alpha+1)=\alpha^{n-3} \cdot \alpha^{3}=\alpha^{n}$.

It follows from the proof of the above theorem that any tree of order $n$ has at most $1.3248^{n}$ minimal 2-dominating sets.

Corollary 3.2. Every tree of order $n$ has at most $\alpha^{n}$ minimal 2-dominating sets, where $\alpha \approx 1.3248$ is the positive solution of the equation $x^{3}-x-1=0$.

Now we show that the bound from the previous corollary is tight. Let $a_{n}$ denote the number of minimal 2-dominating sets of the path $P_{n}$. The next remark follows from the Proof of Theorem 3.1.

Remark 3.3. For every positive integer $n$ we have

$$
a_{n}= \begin{cases}1 & \text { if } n \leq 3 \\ a_{n-3}+a_{n-2} & \text { if } n \geq 4 .\end{cases}
$$

We have $\lim _{n \rightarrow \infty} \sqrt[n]{a_{n}}=\alpha$, where $\alpha \approx 1.3247$ is the positive solution of the equation $x^{3}-x-1=0$. This implies that the bound from Corollary 3.2 is tight.

\section{REFERENCES}

[1] A. Björklund and T. Husfeldt, Inclusion-exclusion algorithms for counting set partitions. Proc. of FOCS (2006) 575-582.

[2] M. Blidia, O. Favaron and R. Lounes, Locating-domination, 2-domination and independence in trees. Australas. J. Combin. 42 (2008) 309-316.

[3] D. Bród and Z. Skupień, Trees with extremal numbers of dominating sets. Australas. J. Combin. 35 (2006) 273-290.

[4] D. Bród, A. Włoch and I. Włoch, On the number of minimal dominating sets including the set of leaves in trees. Internat. J. Contemporary Math. Sci. 4 (2009) 1739-1748.

[5] J.-F. Couturier, P. Heggernes, P. van 't Hof and D. Kratsch, Minimal dominating sets in graph classes: combinatorial bounds and enumeration. Proc. of SOFSEM 2012, Lect. Notes Comput. Sci., vol. 7147. Springer-Verlag, Berlin (2012) 202-213.

[6] D. Eppstein, Small maximal independent sets and faster exact graph coloring, J. Graph Algorithms Appl. 7 (2003) 131-140.

[7] J. Fink and M. Jacobson, n-domination in graphs, Graph Theory with Applications to Algorithms and Computer Science. Wiley, New York (1985) 282-300.

[8] F. Fomin, F. Grandoni, A. Pyatkin and A. Stepanov, Combinatorial bounds via measure and conquer: bounding minimal dominating sets and applications. ACM Transactions on Algorithms, vol. 5, article 9 (2009). 
[9] F. Fomin and D. Kratsch, Exact Exponential Algorithms. Springer, Berlin (2010).

[10] J. Fujisawa, A. Hansberg, T. Kubo, A. Saito, M. Sugita and L. Volkmann, Independence and 2-domination in bipartite graphs. Australas. J. Combin. 40 (2008) 265-268.

[11] T. Haynes, S. Hedetniemi and P. Slater, Fundamentals of Domination in Graphs. Marcel Dekker, New York (1998).

[12] T. Haynes, S. Hedetniemi and P. Slater, Domination in Graphs: Advanced Topics. Marcel Dekker, New York (1998).

[13] M. Kanté, V. Limouzy, A. Mary and L. Nourine, Enumeration of minimal dominating sets and variants, Fundamentals of computation theory. Lect. Notes Comput. Sci., vol. 6914. Springer, Heidelberg (2011) 298-309.

[14] M. Koivisto, An $\mathcal{O}\left(2^{n}\right)$ algorithm for graph coloring and other partitioning problems via inclusion-exclusion. Proc. 47th Annual IEEE Symposium on Foundations of Comput. Sci. (FOCS 2006), IEEE (2006) 583-590.

[15] E. Lawler, A note on the complexity of the chromatic number problem. Information Proc. Lett. 5 (1976) 66-67.

[16] J. Moon and L. Moser, On cliques in graphs. Israel J. Math. 3 (1965) 23-28.

[17] R. Shaheen, Bounds for the 2-domination number of toroidal grid graphs. Internat. J. Comput. Math. 86 (2009) 584-588.

[18] H. Wilf, The number of maximal independent sets in a tree. SIAM J. Algebr. Discrete Methods 7 (1986) 125-130.

Communicated by B. Vallée.

Received May 7, 2012. Accepted April 30, 2013. 